\title{
Observations sur la mise en place du cerne chez le pin d'Alep (Pinus halepensis Mill.) : confrontation entre les mesures de croissance radiale, de densité et les facteurs climatiques
}

\author{
Antoine Nicault*, Cyrille Rathgeber, Lucien Tessier et André Thomas \\ Institut Méditerranéen d’Écologie et de Paléoécologie, CNRS UPRES A 6116, Faculté des Sciences et Techniques Saint Jérôme,
} Case 451, 13397 Marseille Cedex 20, France

(Reçu le 30 août 2000 ; accepté le 14 mai 2001)

\begin{abstract}
Résumé - Afin d'analyser la mise en place du cerne et son déterminisme climatique, quatre pins d'Alep ont fait l'objet d'un suivi en continu de la croissance radiale durant trois années. Les concordances entre les accroissements radiaux, les profils densitométriques des cernes correspondants et les données météorologiques mesurées sur le même site, ont été recherchées. La construction du cerne du pin d'Alep est caractérisée par une phase de croissance printanière, une période de repos estivale et une reprise de croissance à l'automne. La croissance radiale est essentiellement déterminée par la quantité des précipitations du printemps et de l'automne. Les températures interviennent, toujours en combinaison avec les précipitations, sur le démarrage de la croissance, et sur la mobilisation des réserves hydriques estivales. La mise en correspondance des accroissements cumulés et des densitogrammes a permis de dater les variations de densité. La formation du bois initial correspond à l'optimum de la première phase de croissance. À la fin de cette première phase correspond la transition bois initial bois final (juin, juillet). L'alternance repos estival phase de reprise automnale est responsable des fluctuations de densité au sein du bois final.
\end{abstract}

Pinus halepensis / croissance radiale / densité du bois / variations intra-annuelles / cerne double

\begin{abstract}
Intra-annual variations of radial growth and ring structure. Radial growth monitoring of four Aleppo pines is done to analyse ring formation and its climatic determinism. These measures are confronted to wood density profiles and meteorological parameters. Growth occurs in two phases: spring growth and autumnal growth, separated by the summer rest period. The rainfall pattern, before and after the summer drought, plays a major role on ring increment and ring structure. Temperature combined with precipitation, influences growth start and summer water availability. Radial growth measurement and density profiles confrontation makes it possible to associate a temporal scale to density profile. Early wood formation corresponds to optimum of the spring growth phase; early woodlate wood transition corresponds to growth rate reduction at the end of the spring growth phase (June, July); late wood fluctuations are linked with the successive rest period and autumnal growth.
\end{abstract}

Pinus halepensis / intra-annual variations / growth monitoring / wood density / double ring

* Correspondance et tirés-à-part

Tél. : (33) 0491288011 ; Fax : (33) 0491288668 ; e-mail : anicault@yahoo.fr 


\section{INTRODUCTION}

Ce travail s'insère dans une étude dendroécologique plus importante qui a pour objectifs d'analyser les relations production-climat-biotope [34] et de préciser le comportement du pin d'Alep (Pinus halepensis Mill) visà-vis des changements globaux [32]. L'analyse de la relation cerne-climat sur l'ensemble de l'aire de distribution de l'essence, en Provence calcaire, y est abordée par le calcul de fonctions de réponse $[10,43]$. Ce type d'approche statistique portant sur de longues séquences de cernes permet de préciser un comportement moyen de l'arbre vis-à-vis du climat. La croissance radiale annuelle est analysée, non seulement dans ses dimensions globales (épaisseur ou surface du cerne) mais également dans ses dimensions structurelles (densité du cerne). Les paramètres épaisseur et densité, du bois initial et du bois final, fournis par l'analyse densitométrique, traduisent quantitativement les variations intra-annuelles de la structure cellulaire du cerne [31-33,36]. Cependant, l'interprétation des corrélations entre ces paramètres anatomiques, qui apportent une information saisonnière, et les paramètres climatiques reste, en l'absence d'informations précises sur les mécanismes physiologiques de mise en place du cerne, spéculative et le plus souvent éloignée des relations de cause à effet.

Ce travail fait suite à des études déjà réalisées sur la construction du cerne du pin d'Alep, en Israël [25-27], au Maroc [1], en Italie [2] et en France [39, 40, 42].

Le travail présenté ici, focalisé sur les variations intraannuelles de croissance et de densité, a pour objectif de fournir des éléments d'interprétation des relations cerne-climat mises en évidence par l'approche statistique. L'objectif est également de définir, par rapport aux différentes étapes de la mise en place du cerne, les paramètres de densité utilisables en dendroécologie et dendroclimatologie. Cette approche permettra également d'aborder le phénomène de formations des cernes doubles fréquemment relevés en dendrochronologie $[4,10$, 35].

Notre étude, effectuée sur un seul site, et quatre arbres, met en œuvre deux approches. L'enregistrement simultané des accroissements radiaux et des paramètres climatiques permet de suivre pas à pas l'élaboration du cerne en relation avec les conditions climatiques locales. L'analyse densitométrique d'éprouvettes de bois prélevées, dans un second temps, sur chaque arbre fournit une caractérisation structurelle du cerne. La confrontation des deux types de données permet d'appréhender, pour chaque année et chaque arbre, les relations climat-croissance-structure du cerne.

\section{MATÉRIEL ET MÉTHODES}

Quatre pins d'Alep, provenant d'un même site d'étude, ont fait l'objet d'un suivi en continu de la croissance radiale et d'une analyse densitométrique a posteriori. La présente étude ne porte que sur quatre pins car nous ne disposions que d'un enregistreur six canaux pour ce dispositif expérimental; deux chênes verts (Quercus ilex L.) ont également fait l'objet de mesures. Les mesures ont été réalisées sur trois ans (1993, 1994, et 1995).

\subsection{Site d'étude}

Le site d'étude est localisé sur la commune de Gardanne $\left(5^{\circ} 29^{\prime} \mathrm{E}, 43^{\circ} 28^{\prime} \mathrm{N}\right)$ à une altitude de $230 \mathrm{~m}$ (figure 1). Il est soumis à un climat de type méditerranéen caractérisé une période de sécheresse estivale bien marquée (figure 2).
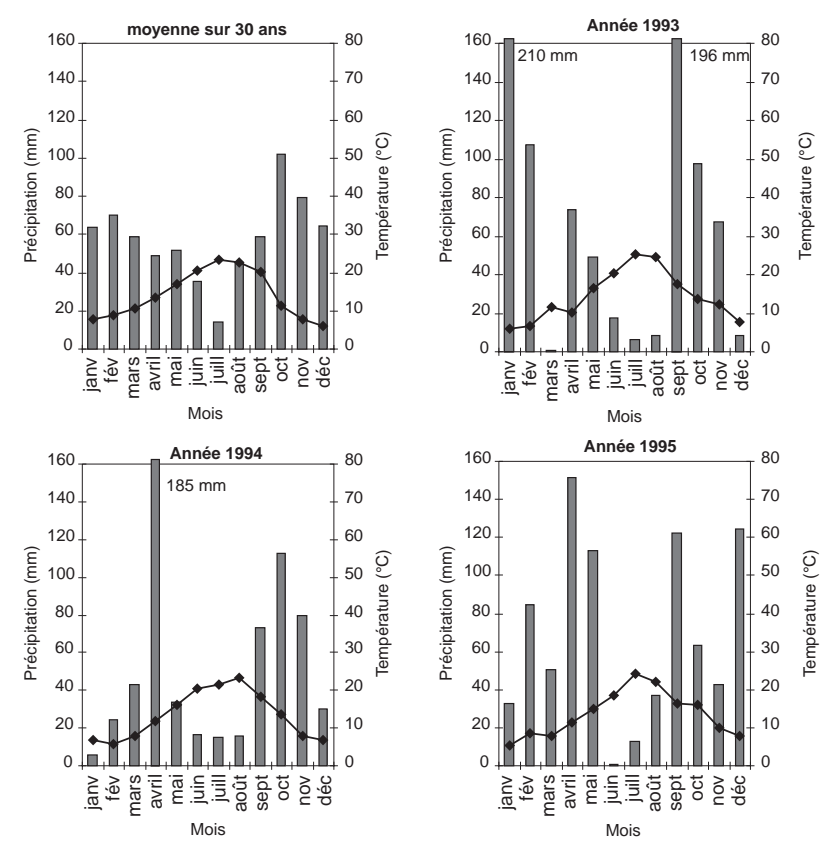

Figure 1. Localisation du site. 

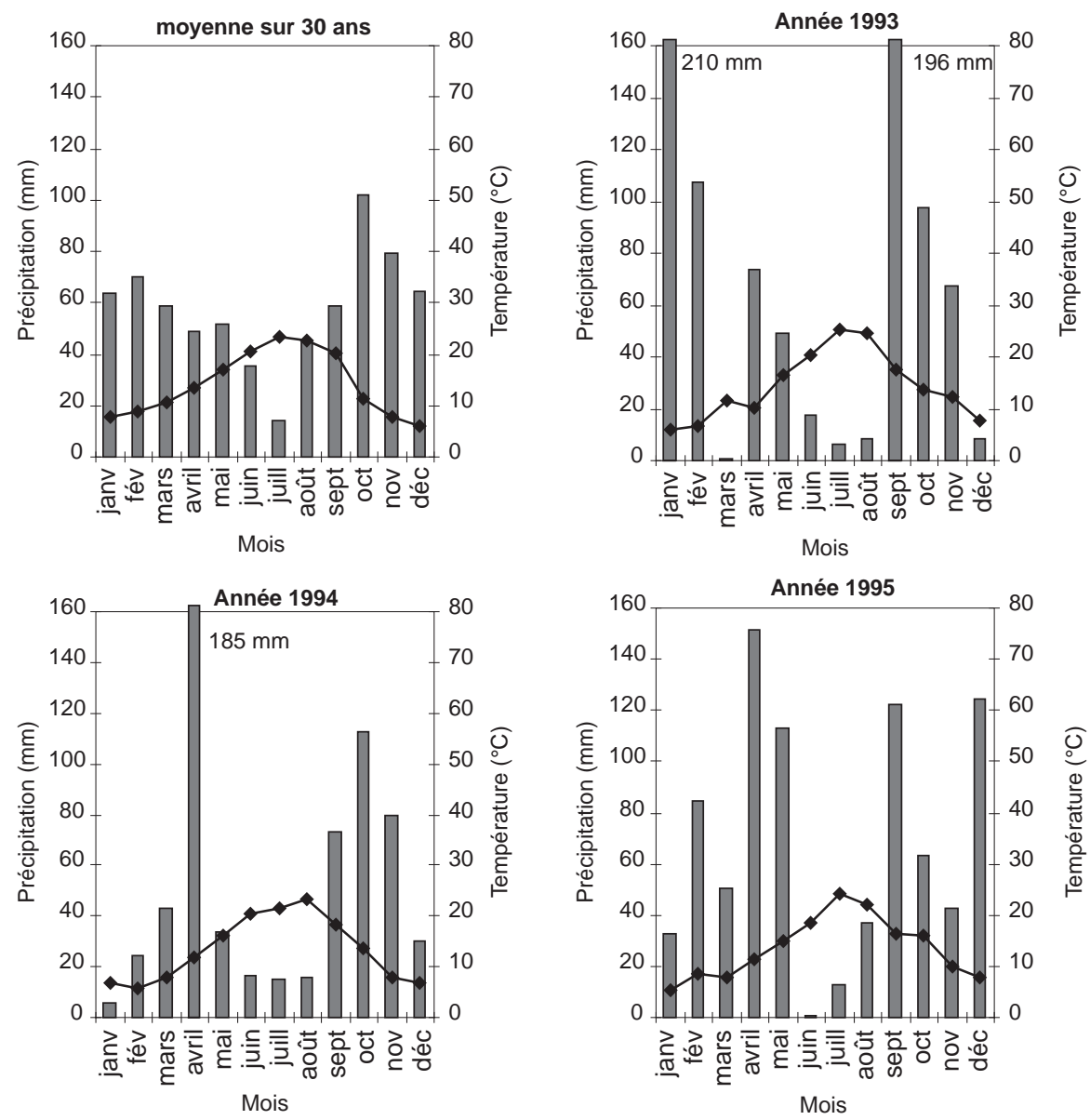

Figure 2. Diagrammes ombrothermiques effectués à partir de la moyenne des précipitations $(\mathrm{mm})$ et des températures de la période (1961-1990) et des températures et précipitations des trois années analysées : 1993, 1994 et 1995. (Station Météo-France de Gardanne).

Le site est en exposition ouest avec une pente de $5 \%$. Le sol d'une profondeur d'environ 60 à $70 \mathrm{~cm}$ est composé de $30 \mathrm{~cm}$ de colluvion puis d'une altérite de $30 \mathrm{~cm}$. Le colluvium a une texture limono-sableuse et est moyennement chargé en éléments grossiers $(<20 \%)$; l'altérite présente une texture limono-argilo-sableuse et est fortement chargée en éléments grossiers (> $60 \%$ ). La roche sous-jacente, enfin, est de type calcaire dur.

Les quatre pins d'Alep choisis sont dominants ou codominants et présentent un bon état sanitaire. Ils sont répartis sur une surface boisée d'environ $1000 \mathrm{~m}^{2}$. Aucune information n'est disponible en ce qui concerne les activités anthropiques ou les incendies qui auraient éventuellement affecté le peuplement (aucunes marques ou cicatrices ne sont visibles sur les arbres). Actuellement, le peuplement ne fait l'objet d'aucun traitement sylvicole.

Les arbres analysés ont été choisis lors du protocole expérimental d'âges différents (tableau I) et avec une position différente au sein du peuplement, afin de représenter l'ensemble des individus présents sur les sites. Pinus 1 (P1) et Pinus 2 (P2) se situent en lisière du peuplement et peuvent, actuellement, presque être considérés comme des arbres isolés, tandis que les deux autres pins (Pinus 3 (P3) et Pinus 4 (P4)), dominants, et se trouvent au sein du peuplement qui est assez dense. Ce peuplement est essentiellement composé de Pinus halepensis, Quercus ilex, et Quercus pubescens. La

Tableau I. Âge (en années, à 1,30 m) et circonférence (cm) à $1,30 \mathrm{~m}$ des arbres mesurés ; position au sein du peuplement.

\begin{tabular}{lccl}
\hline Arbre & Age & Circonférence & Position/peuplement \\
\hline Pinus 1 & 90 & 185 & Lisière \\
Pinus 2 & 30 & 98 & Lisière \\
Pinus 3 & 24 & 40 & Couvert \\
Pinus 4 & 35 & 60 & Couvert \\
\hline
\end{tabular}


strate arbustive est composée principalement de Quercus coccifera, Phillyrea angustifolia, et Rhamnus alaternus et la strate herbacée principalement de Brachypodium retusum, Brachypodium phoenicoïdes et Asparagus acutifolius.

Sur le même site sont récoltées les données croissance et les données météorologiques.

\subsection{Données croissance}

Un historique des méthodes utilisées pour suivre l'élaboration des cernes de croissance est présenté dans les travaux de Serre [39]. Dans le cadre de notre recherche, la station de mesure a été élaborée d'après un modèle créé par Kurt Loris [28, 29]. Les dendrographes utilisés sont des capteurs électroniques à résistance (potentiomètre linéaire MEGATRON (Müchen) 1000 ohms) appliqués ponctuellement sur le tronc à $1,30 \mathrm{~m} \mathrm{du}$ sol (niveau de prélèvement en dendroécologie). Un capteur a été placé sur chaque arbre. Avant de positionner le capteur, l'écorce est enlevée au point de contact afin de minimiser les variations en épaisseur induites par le fonctionnement de l'assise subero-phellodermique. Le capteur est placé sur un support fixé, sur les cotés du tronc, à l'aide de deux pattes métalliques vissées profondément dans le tronc. Aucun traumatisme important, susceptible de perturber les enregistrements, n'a été relevé. Les capteurs réagissent à des variations du rayon du tronc de l'ordre du 1/100 mm. Ils sont calibrés une première fois avant le début de la période de végétation et une seconde fois au début de l'été.

Le déplacement linéaire, amplifié, de chaque capteur est transmis toutes les minutes à un enregistreur (type LICOR) qui mémorise la valeur moyenne horaire.

Les enregistrements des variations du rayon ont été effectués en continu sur la période 15 avril 1993-20 octobre 1995. Ils sont représentés, pour chaque année et chaque arbre, par des courbes d'accroissements (horaires ou quotidiens) cumulés et par des histogrammes représentant les taux de croissance sur quinze jours. La discontinuité des courbes d'accroissements cumulés est la conséquence de problèmes techniques liés à des incidents d'alimentation du système.

\subsection{Données radiodensitométriques}

Les cernes, dont la croissance a été suivie pendant trois ans, ont été prélevés au point de mesure (carottage à la tarière de Pressler) l'année suivant l'arrêt définitif de la station d'enregistrement. Les quatre échantillons ont fait l'objet d'une analyse densitométrique dont les techniques récentes, mises en œuvre ici, sont détaillées par Schweingruber [36, 37]. La majeure partie de la préparation des échantillons et la réalisation des clichés radiographiques a été effectuée avec la collaboration du W.S.L. de Birmensdorf et du Centre d'Etude Nordique de l'Université Laval de Québec. Après extraction des résines, des sucres, et des tanins, plus ou moins opaques aux rayons $\mathrm{X}$, les carottes sont découpées en lames d'épaisseur calibrée $(1,2 \mathrm{~mm}$, avec 1 à $2 \%$ d'erreur maximum). Ces dernières sont ensuite exposées, environ une heure, sous une source de rayons $\mathrm{X}$ faiblement énergétiques $(11 \mathrm{kV}, 20 \mathrm{~mA})$. Les radiographies obtenues sont analysées au moyen d'un microdensitomètre optique (Walesch Electronic). Les mesures effectuées permettent d'obtenir le profil détaillé des variations de densité du cerne (densitogramme) pour chaque année et chaque arbre.

Il existe de nombreuses définitions de la limite bois initial-bois final [8, 14, 21]. Dans le cadre de l'étude, la limite bois initial-bois final a été déterminée par la méthode de la limite flottante qui reflète beaucoup mieux la biologie de l'arbre qu'une limite bois initial-bois final fixe quels que soient les cernes $[14,36]$. La limite a été choisie conventionnellement à $50 \%$, soit :

$$
D_{\lim }=\frac{D_{\max }+D_{\min }}{2}
$$

avec $D_{\min }$ : densité minimale du cerne, $D_{\max }$ : densité maximale du cerne.

\subsection{Données climatiques}

La station météorologique est implantée sur le site d'étude hors couvert forestier, à une cinquantaine de mètre du peuplement. Les précipitations $(\mathrm{mm})$, la température $\left({ }^{\circ} \mathrm{C}\right)$, et 1 'hygrométrie relative $(\%)$ sont enregistrées à un pas de temps semi-horaire. Les lacunes (période hivernale précédant l'installation de la station et trois semaines durant le mois de janvier 1995) sont comblées grâce aux données quotidiennes recueillies par le poste d'Aix-en-Provence (Météo-France). Celles de la station de Gardanne n'étaient pas disponibles dans le cadre de l'étude. Cependant, la comparaison des données mensuelles des postes d'Aix-en-Provence et de Gardanne montre que l'ampleur et l'intensité des variations sont similaires d'un poste à l'autre.

La somme des précipitations mensuelles et les températures moyennes mensuelles sont calculées afin d'avoir 


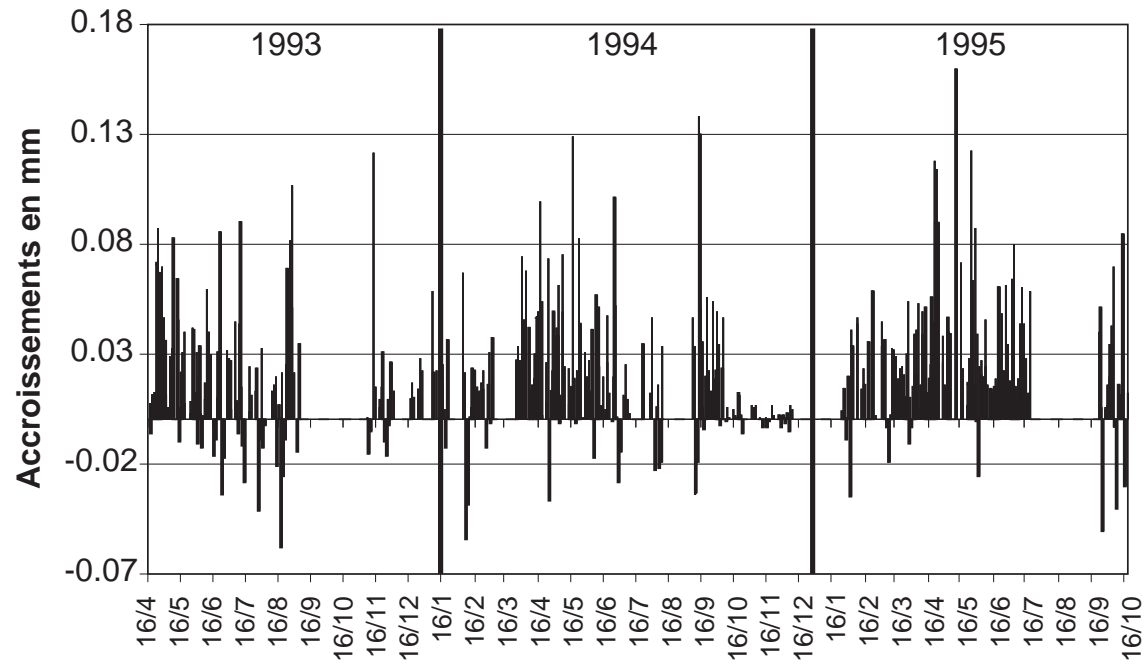

Figure 3. Taux de croissance en rayon quotidiens $(\mathrm{mm})$ calculés à partir des mesures en continu de la croissance de Pinus 2. une représentation synthétique des caractéristiques météorologiques des trois années étudiées. Elles sont représentées sous forme de diagrammes ombrothermiques et confrontées au diagramme ombrothermique effectué sur la base d'une moyenne de trente ans calculée sur la période 1961-1990. Les diagrammes sont effectués à partir des données fournies par la station Météo-France de Gardanne.

L'utilisation des données météorologiques est effectuée au niveau mensuel, aussi bien qu' au niveau journalier, voire intra-journaliers en ce qui concerne les gelées matinales (considérées quand la température minimale est inférieure à $0{ }^{\circ} \mathrm{C}$ ). Le nombre de jours présentant une température moyenne inférieure à $4{ }^{\circ} \mathrm{C}$ est également utilisé pour la période hivernale. En effet, d'après Serre [39], des températures inférieures à $4{ }^{\circ} \mathrm{C}$ sont susceptibles de retarder le démarrage de la croissance.

\subsection{Méthodes d'analyse}

Le petit nombre d'années et d'individus mesurés ne permettent pas d'appliquer des méthodes statistiques; l'analyse relève donc du seul domaine de l'observation. En effet, seul le calcul de taux de croissance quotidiens aurait pu fournir assez de données pour effectuer une analyse statistique entre données croissance et données climatiques. Mais l'utilisation des taux de croissance quotidiens (figure 3) s'est révélée peu pertinente compte tenu des artefacts que représentent les fluctuations du rayon associées aux mouvements de l'eau dans le tronc (figure 4B) [12, 30, 44]. Aucune des techniques testées n'a pu, de façon satisfaisante, extraire les signaux dus à la croissance quotidienne de l'ensemble des autres signaux.

Les relations entre le climat et la construction du cerne sont donc recherchées par la simple observation des concordances entre la dynamique de la croissance radiale, les fluctuations de densité au sein du cerne et les facteurs météorologiques disponibles à partir des données collectées sur le site. La confrontation des profils de densité aux courbes d'accroissements cumulés et aux taux de croissance respectifs s'effectue sur leur seule base commune: l'épaisseur du cerne. Des différences d'épaisseur de cernes, observées entre les épaisseurs relevées sur les profils et celles relevées sur les courbes de croissance en continu, ont nécessité un ajustement graphique.

\section{RÉSULTATS}

\subsection{Croissance}

L'observation des courbes de croissance (figure 4) montre que les arbres les plus jeunes (P2, P3, P4) ont une croissance plus importante que l'arbre le plus vieux (P1) et que Pinus 2, en lisière du peuplement (tableau I) a une croissance plus importante que les deux arbres poussant au sein du peuplement (P2, P3).

L'analyse des accroissements cumulés (figure 4) et des taux de croissance (figure 5), tout au long de la période de végétation, permet de préciser, pour chaque arbre et chaque année, les différentes phases d'activité 


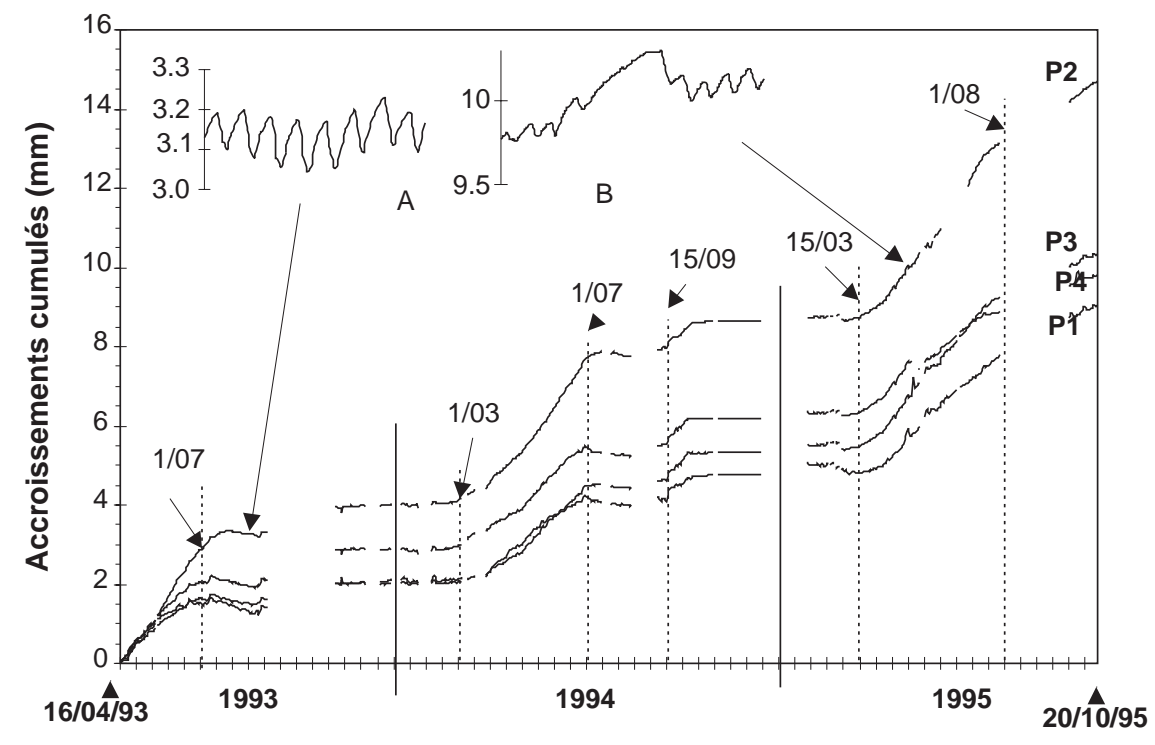

Figure 4. Courbes des accroissements radiaux quotidiens cumulés $(\mathrm{mm})$ de P1, P2, P3, P4, pour les trois années 1993, 1994 et 1995. A : Zoom sur les fluctuations nycthémérales du rayon du tronc. B : Zoom sur la perturbation des fluctuations nycthémérales du tronc par l'occurrence de précipitations.

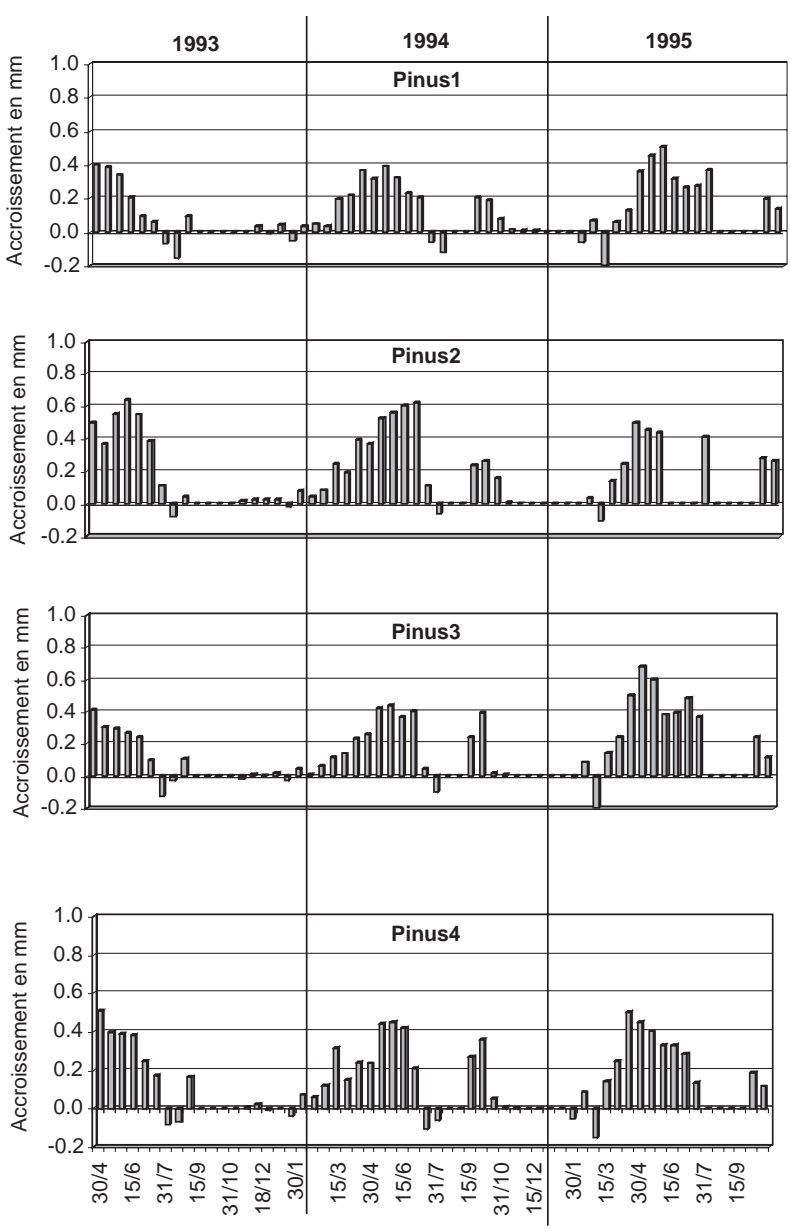

Figure 5. Taux de croissance en rayon calculés sur la base de périodes de 15 jours. cambiale (tableau II). La période d'activité cambiale dure de sept à huit mois. Elle est divisée, par une période de repos durant l'été, en deux phases de croissance distinctes ; la première au printemps et la seconde à l'automne (visibles les années 1993 et 1994). La première phase représente entre 80 et $90 \%$ de la croissance totale. En 1995, en raison des problèmes d'enregistrement, la présence d'une période de repos estival ne peut être affirmée avec certitude. Cependant, une diminution importante de la croissance, plus tardive que lors des deux autres années, peut être notée durant le mois d'août. À partir de ce modèle commun de croissance, des variations, inter-individus et inter-annuelles, des rythmes et des taux de croissance sont observées.

Pour une année donnée, un décalage de 10 à 15 jours entre les individus est observé pour les dates de démarrage de la croissance et de diminution, ou de mise en repos, de la croissance estivale (tableau II). En revanche, la reprise de croissance automnale (seulement identifiable pour l'année 1994) est synchrone pour l'ensemble des individus. Des variations inter-individuelles sont également observées au niveau taux de croissance sur la phase printanière.

Les variations inter-annuelles du rythme de croissance concernent l'ensemble des étapes de la mise en place du cerne. On observe un décalage de plus d'un mois entre les dates de démarrage de la croissance, avec notamment en 1994, un démarrage précoce à la fin du mois de février. Les périodes de diminution de la croissance, correspondant à la mise en repos estivale, révèlent 
Tableau II. Dates et durées des différentes phases de croissance observées (j : jours).

\begin{tabular}{|c|c|c|c|c|c|c|c|c|}
\hline \multirow[b]{3}{*}{ Années } & \multicolumn{5}{|c|}{ Calendrier } & \multicolumn{3}{|c|}{ Durée } \\
\hline & \multicolumn{8}{|c|}{ Période d'enregistrement : 16/04/1993 au 20/10/1995 } \\
\hline & Arbres & Départ & Repos & Reprise & Arrêt & Phase1 & Repos & Phase 2 \\
\hline \multirow[t]{4}{*}{1993} & $\mathrm{P} 1$ & $22 / 4$ & $12 / 7$ & - & - & - & - & - \\
\hline & P2 & \multirow{3}{*}{ 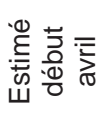 } & $27 / 7$ & - & - & - & - & - \\
\hline & P3 & & $12 / 7$ & - & - & - & - & - \\
\hline & P4 & & $12 / 7$ & - & - & - & - & - \\
\hline \multirow[t]{4}{*}{1994} & $\mathrm{P} 1$ & $1 / 3$ & $30 / 6$ & $14 / 9$ & $6 / 10$ & $122 \mathrm{j}$ & 75 j & 22 j \\
\hline & P2 & $23 / 2$ & $12 / 7$ & $14 / 9$ & $5 / 10$ & $143 \mathrm{j}$ & $61 \mathrm{j}$ & $21 \mathrm{j}$ \\
\hline & P3 & $1 / 3$ & $12 / 7$ & $14 / 9$ & $3 / 10$ & $134 \mathrm{j}$ & $72 \mathrm{j}$ & $20 \mathrm{j}$ \\
\hline & P4 & $23 / 2$ & $30 / 6$ & $14 / 9$ & $3 / 10$ & $129 \mathrm{j}$ & $61 \mathrm{j}$ & $20 \mathrm{j}$ \\
\hline \multirow[t]{4}{*}{1995} & P1 & - & \multirow{4}{*}{ 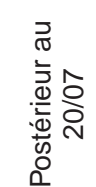 } & - & \multirow{4}{*}{ 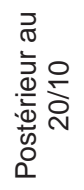 } & - & - & - \\
\hline & P2 & $13 / 3$ & & - & & - & - & - \\
\hline & P3 & - & & - & & - & - & - \\
\hline & P4 & $10 / 3$ & & - & & - & - & - \\
\hline
\end{tabular}

également le même type de décalage (de mi-juin à fin juillet selon les années).

L'analyse des taux de croissance est à considérer avec précautions car, bien que les fluctuations du tronc liées aux mouvements d'eau soient atténuées, elles n'en restent pas moins présentes. En effet, ceci est bien visible à travers la présence de taux de croissance négatifs ( $f i$ gure 5) en juillet. Ces valeurs négatives sont liées au phénomène de rétraction du tronc durant la période sèche.

Cependant, l'analyse des taux de croissance, calculés sur des périodes de quinze jours, semble mettre en évidence les phases d'accélération ou de ralentissement de la croissance radiale (figure 5). Ces phases varient également d'une année sur l'autre. En 1993, le démarrage de la croissance est brutal puis la croissance ralentit progressivement ; en 1994, le démarrage est progressif puis suivi d'une diminution plus brutale ; en 1995 des accélérations de croissance se produisent dans la seconde partie de la phase printanière (juin).

\subsection{Fluctuations de densité}

La structure générale du cerne de pin d'Alep présente schématiquement deux zones : le bois initial qui a une densité variant, en moyenne, de 350 à $550 \mathrm{~kg} / \mathrm{m}^{3}$, et le bois final qui a une densité variant, en moyenne, de 550 à $750 \mathrm{~kg} / \mathrm{m}^{3}$. Les profils de densité (figure 6 ) montrent que le type de transition entre le bois initial et le bois final est variable. Elle est vraiment graduelle pour Pinus 4 et pour tous les arbres en 1995. Pour les trois autres pins, une rupture plus ou moins nette est observée selon les années.

Cette rupture est précédée, en 1993 et 1994, d'une augmentation de la densité du bois initial qui semble indépendante de la transition bois initial-bois final. En 1995, on peut également relever au sein du bois initial un type de fluctuation de la densité plus réduit en intensité et plus ponctuel, qui affecte (de façon plus ou moins évidente) l'ensemble des individus (figure 6).

Les variations de densité au sein du bois final des cernes de Pinus 1 (1993) et de Pinus 4 (1994) fournissent deux profils, qui peuvent être considérés comme archétypes, pour les quatre arbres mesurés (figure 6). Le premier est caractérisé par une faible épaisseur de bois final et un seul pic de densité, le deuxième par un bois final nettement plus important et une diminution de la densité entre deux pics de forte densité. Les autres profils sont des intermédiaires entre ces deux types. Les profils des arbres $\mathrm{P} 1$ et $\mathrm{P} 4$, très différents, peuvent s'expliquer par une différence d'âge très importante entre les deux individus. Les cernes de $\mathrm{P} 1$ ont un âge cambial supérieur à 

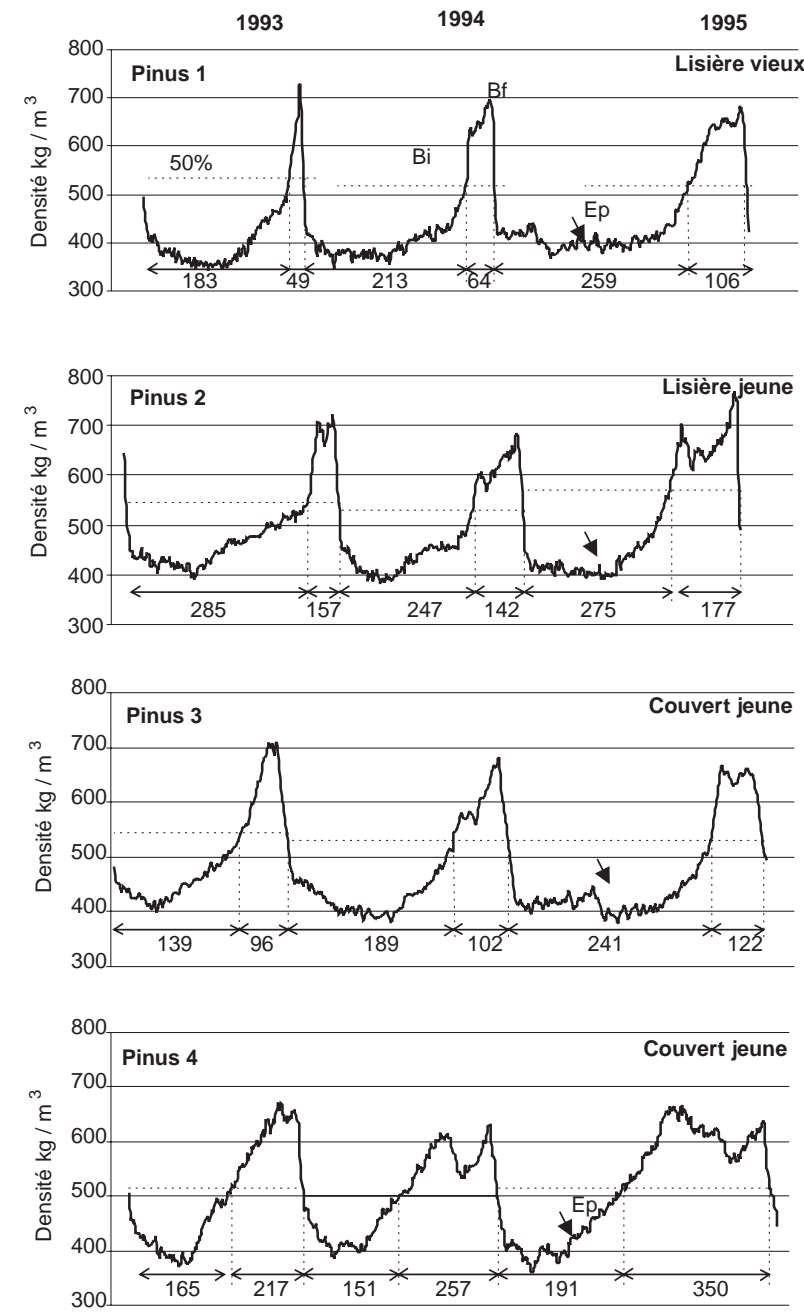

Figure 6. Profils intra-annuels de densité (densitogrammes) de P1, P2, P3, P4 pour les trois années 1993, 1994 et 1995. Les épaisseurs du bois initial (Bi) et du bois final (Bf) sont notées en $1 / 100 \mathrm{~mm}$.

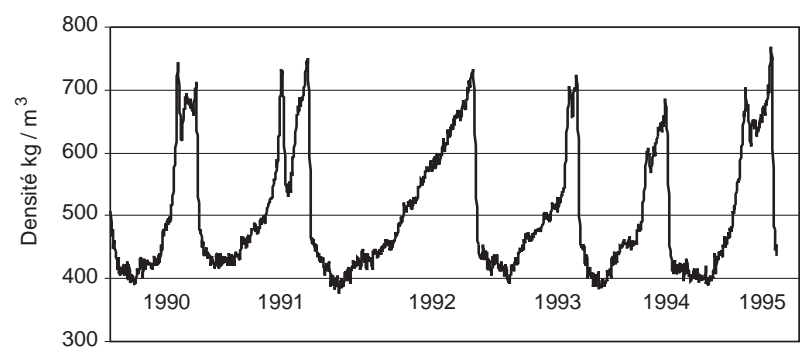

Figure 7. Densitogrammes des années 1990 à 1995 (Pinus 2). Mise en évidence de la variété des densitogrammes sur une courte période. quatre-vingt cinq ans, alors que les cernes de P4 sont âgés d'environ 30 ans.

Il faut cependant noter que les douze profils étudiés ne représentent que partiellement la variation importante des profils de densité relevés au sein des cernes de pin d'Alep. Les profils de densité, de Pinus 2, des années 1990 à 1995 expriment mieux cette variabilité (figure 7).

\subsection{Relation densité - croissance}

Les correspondances notées entre les courbes d'accroissements cumulés et les densitogrammes des cernes correspondants (figure 8) donnent une idée assez juste des différentes phases de la formation du cerne et de leurs relations avec sa structure, pour les trois années mesurées. D'après l'ajustement graphique des deux types de données, le bois initial est formé durant la période comprenant les mois de mars à mai ; la transition entre bois initial et bois final s'effectue au mois de juin ; le bois final est construit durant la période comprenant les mois de juillet à octobre. Les deux phases de croissance printanière et automnale ne correspondent pas respectivement au bois initial et au bois final. Le bois initial semble être entièrement construit pendant la première partie de la croissance printanière, c'est-à-dire durant la phase d'augmentation puis de stabilisation des taux de croissance. La construction du bois final semble commencer avec la diminution des taux de croissance à la fin de la phase de croissance printanière, et se poursuit jusqu'à la fin de la période de végétation. La période estivale n'a donc une influence que sur la structure du bois final.

Les fluctuations importantes des taux de croissance se répercutent de façon inverse au niveau des variations de densité: une augmentation des taux de croissance concorderait avec une diminution de la densité, et inversement une diminution des taux de croissance correspondrait à une augmentation de la densité. En 1993, l'augmentation progressive de la densité du bois initial avant la transition nette entre bois initial et bois final semble correspondre à une diminution progressive des taux de croissance précédant leur diminution brutale.

Les diminutions de densité au sein du bois final, observables sur certains des profils, sont associées à la reprise de croissance qui fait suite à la période estivale. L'arrêt définitif de la croissance, précédé d'une diminution brutale des taux de croissance, correspond à la formation du deuxième pic de densité, généralement plus élevé que le premier, qui marque la fin du cerne. En 1993, le bois final de Pinus 1 n'est caractérisé que par un seul pic de densité (figure 6), aucune fluctuation de densité 

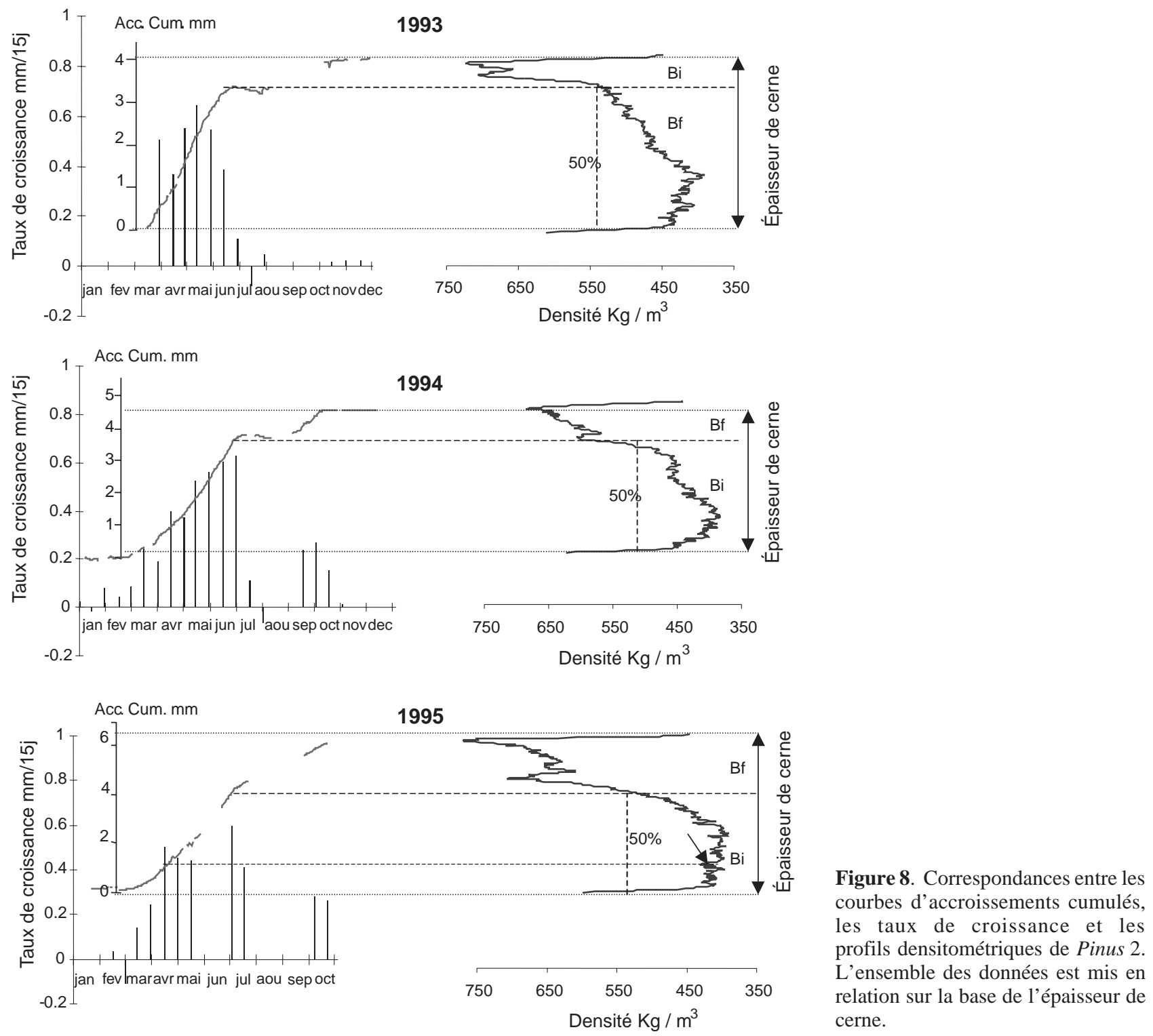

n'est décelable et cependant la courbe d'accroissement cumulé correspondante (figure 4) montre une période de repos. Cette observation permet de mettre en doute l'arrêt total de la croissance pendant la période estivale. La période de repos observée ne correspondrait, dans ce cas, qu'à une forte diminution de la croissance.

\subsection{Les relations climat - croissance - densité du bois}

En 1993, la somme des précipitations (635 mm) est nettement inférieure à la somme des précipitations des années 1994 et 1995 (respectivement $842 \mathrm{~mm}$ et $835 \mathrm{~mm}$ ). La somme des précipitations de l'année 1993 est légèrement inférieure à la moyenne des 50 dernières années, tandis que les sommes des précipitations des années 1994 et 1995 sont nettement supérieures. Les précipitations annuelles des trois années se différencient par leur abondance, mais surtout par leur répartition au cour de l'année (figure 2). Les températures moyennes annuelles des trois années, $13,4{ }^{\circ} \mathrm{C}$ en $1993,14,3{ }^{\circ} \mathrm{C}$ en 1994 et $13,5^{\circ} \mathrm{C}$ en 1995 sont toutes inférieures à la moyenne des trente dernières années 
$\left(15^{\circ} \mathrm{C}\right)$. L'évolution des températures est similaire pour les trois années et ne présente aucun événement remarquable. Les trois années 1993, 1994, et 1995 possèdent une période de sécheresse estivale $(\mathrm{P}<2 \mathrm{~T})$ d'environ trois mois supérieure à la moyenne (2 mois) (figure 2$)$. Cependant les périodes de sécheresse des ces trois années ne sont pas exceptionnelles pour la région, d'autant plus que les fortes précipitations orageuses de la fin du mois d'août perturbent quelque peu la moyenne.

\subsubsection{Le démarrage de la croissance}

Aucune relation n'est observée entre les températures moyennes mensuelles des mois de janvier, février, mars et les dates de démarrage de la croissance (tableau III). En revanche, la confrontation des dates de démarrage avec le nombre de jours de gelée matinale et le nombre de jours où la température moyenne journalière est inférieure à $4{ }^{\circ} \mathrm{C}$, montre des concordances. Le démarrage tardif de la croissance en 1993, concorde avec un mois de février froid, comportant des minima très accusés $\left(-8^{\circ} \mathrm{C}\right)$ et, début mars, trois jours en dessous de $4{ }^{\circ} \mathrm{C}$. Le démarrage précoce de la croissance en 1994 coïncide avec un mois de février doux qui ne comporte que quatre jours avec présence de gelées matinales. Cependant, en 1995, malgré un mois de février très doux, on observe un décalage de quinze jours, du démarrage de la croissance, avec l'année 1994. Ce décalage semble coïncider avec des mois de janvier et février beaucoup plus secs en 1995 qu'en 1994 (tableau III).

\subsubsection{Le comportement estival}

Les dates de ralentissement de la croissance sont confrontées aux sommes des précipitations des mois d'avril-mai, et de juin-juillet, ainsi qu' aux températures moyennes de juin et juillet pour chacune des trois années (tableau III). Les dates de ralentissement de la croissance concordent avec la somme des précipitations de la période avril-mai. Plus les précipitations sont importantes

Tableau III. Dates de démarrage de la croissance et caractéristiques météorologiques des mois de janvier, février, mars des années 1993, 1994 et 1995 (* minimum absolu relevé sur le site); dates de mise en repos et caractéristiques météorologiques des périodes avril mai et juin - juillet.

\begin{tabular}{|c|c|c|c|c|c|c|c|}
\hline Événement & Dates & Années & Mois ou période & $\begin{array}{c}\text { Nbre de jours } \\
\text { Tmoy }<4^{\circ} \mathrm{C}\end{array}$ & $\begin{array}{l}\text { Nbre de jours } \\
\text { avec gelées }\end{array}$ & Tmoy $\left({ }^{\circ} \mathrm{C}\right)$ & $P(\mathrm{~mm})$ \\
\hline \multirow{9}{*}{ 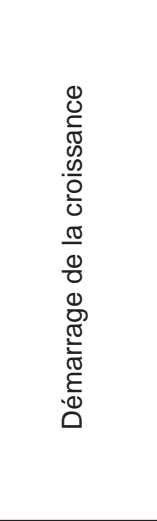 } & \multirow{3}{*}{ Estimé mi avril } & \multirow{3}{*}{1993} & Janvier & 5 & $?$ & 6,99 & 5,6 \\
\hline & & & Février & 7 & $12\left(-8.2^{\circ} \mathrm{C}\right)^{*}$ & 5,61 & 24,5 \\
\hline & & & Mars & 3 & 0 & 7,91 & 42,7 \\
\hline & \multirow{3}{*}{ Du $23 / 2$ au $1 / 3$} & \multirow{3}{*}{1994} & Janvier & 7 & 0 & 5,99 & 210,0 \\
\hline & & & Février & 3 & $4\left(-1.9^{\circ} \mathrm{C}\right)^{*}$ & 6,53 & 107,0 \\
\hline & & & Mars & 0 & 0 & 11,45 & 1,0 \\
\hline & \multirow{3}{*}{ Du 10/03 au 13/03 } & \multirow{3}{*}{1995} & Janvier & 13 & $?$ & 5,30 & 32,4 \\
\hline & & & Février & 0 & 0 & 8,53 & 84,6 \\
\hline & & & Mars & 2 & 0 & 7,73 & 50,8 \\
\hline \multirow{6}{*}{ 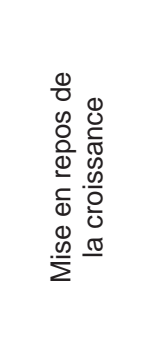 } & \multirow{2}{*}{ Du $12 / 7$ au $27 / 7$} & \multirow{2}{*}{1993} & Avril-Mai & 0 & 0 & 14,30 & 219,0 \\
\hline & & & Juin-Juillet & 0 & 0 & 21,09 & 31,9 \\
\hline & \multirow{2}{*}{ Du 30/06 au 12/6 } & \multirow{2}{*}{1994} & Avril-Mai & 0 & 0 & 13,23 & 123,0 \\
\hline & & & Juin-Juillet & 0 & 0 & 22,72 & 24,0 \\
\hline & \multirow{2}{*}{ Après le 20/07 } & \multirow{2}{*}{1995} & Avril-Mai & 0 & 0 & 13,05 & 265,0 \\
\hline & & & Juin-Juillet & 0 & 0 & 21,31 & 13,0 \\
\hline
\end{tabular}


durant cette période plus la date de mise en repos est retardée. En revanche, aucune relation entre la somme des précipitations de la période juin-juillet et la mise en repos n'a pu être mise en évidence. Il faut cependant noter que les variations des précipitations d'une année sur l'autre de la période juin-juillet sont bien moins importante que pour la période avril-mai.

En 1994, la température moyenne de la période juin-juillet, la plus élevée, correspond à l'année où la mise en repos est la plus précoce.

\subsubsection{La croissance automnale}

La reprise de croissance à l'automne n'est observable avec certitude que pour l'année 1994. La reprise, synchrone pour tous les individus, concorde avec les premières fortes précipitations qui suivent la période de sécheresse (figure 9). Le fait que le phénomène de croissance ne soit nettement identifiable qu'à partir du 24 septembre ne présume en rien d'un démarrage de processus cellulaires qui interviendraient dès le début de la restauration du stock d'eau (vers le 14 septembre). L'occurrence de précipitations à l'issue de la période de sécheresse apparaît donc, à travers nos enregistrements, comme le facteur déterminant de la reprise automnale. Cette dernière résulterait donc d'un événement météorologique ponctuel.

\subsubsection{L'arrêt de la croissance}

L'arrêt de la croissance n'est observable que pour l'année 1994. Le manque de données ne nous permet donc pas d'identifier de façon précise une relation entre

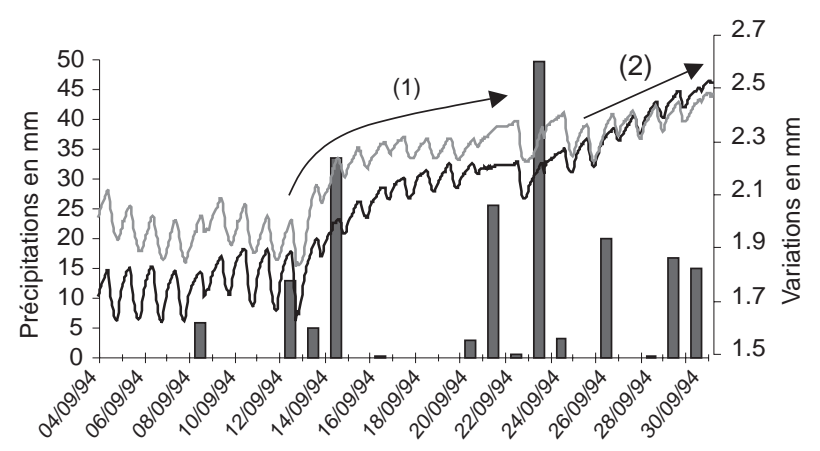

Figure 9. Influence des précipitations (cumul journalier en mm) sur la reprise de croissance (accroissements horaires cumulés mm) de Pinus 2 et Pinus 3 faisant suite à la période de repos estivale; (1) Restauration du stock d'eau; (2) Croissance automnale. les variables météorologiques et l'arrêt de l'activité cambiale.

\subsubsection{Les fluctuations de densité}

La confrontation des profils de densité aux courbes d'accroissements cumulés permet d'associer les fluctuations ponctuelles de la densité, observées au sein du bois initial des cernes de l'année 1995, aux mois d'avril et mai (figure 8). Ces chutes ponctuelles de densité concordent avec l'occurrence de précipitations importantes (entre 40 et $50 \mathrm{~mm}$ ) (figure 10).

Les variations au sein du bois final peuvent être mises en parallèle avec les précipitations de la fin de l'été. En effet, ces dernières étant responsables de la reprise de croissance à l'automne, elles sont par voie de fait à l'origine des fluctuations de densité au sein du bois final.

La transition bois initial-bois final correspond toujours à la conjugaison d'une forte diminution des précipitations et à une augmentation des températures. La combinaison de ces deux facteurs conduit à un bilan hydrique négatif et donc à un stress hydrique.

\section{DISCUSSION}

Outre le fait que les quatre pins étudiés sur trois années 1993, 1994 et 1995 ne représentent que très partiellement les multiples combinaisons envisageables, la méthode de suivi en continu de croissance utilisée et surtout la méthode utilisée quant à la mise en relation dynamique de croissance et structure du cerne soulève certains problèmes.

L'utilisation d'un seul capteur par arbre ne prend pas en compte les variations, qui peuvent être importantes, selon le rayon du tronc [18, 48]. De plus, le calendrier de croissance établi à partir des courbes d'accroissements cumulés ne correspond pas précisément au calendrier de l'activité cambiale, mais, en dehors des phénomènes de turgescence, à celui des seuls phénomènes de croissance observables, associés à la division et à l'élongation cellulaire. En effet, le réveil de l'activité cambiale est antérieur à toute division cellulaire [39, 47]. De la même façon, l'arrêt de la croissance enregistré par les capteurs ne veut pas dire qu'il y a arrêt de l'activité cambiale et mise en repos. Seuls des prélèvements de la zone cambiale pourraient nous informer sur les rythmes précis de l'activité cambiale. De plus, les phénomènes de lignification des parois peuvent durer jusqu'à deux ou trois mois 

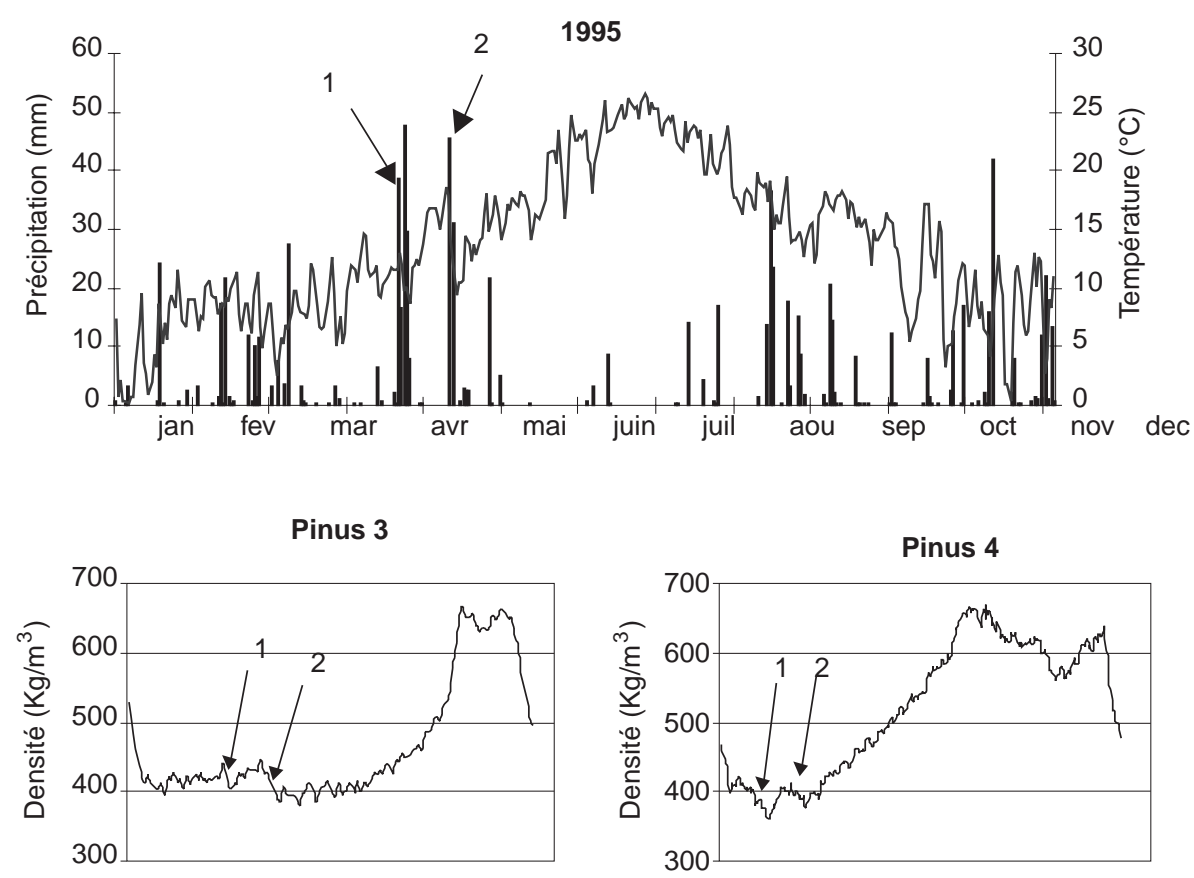

Figure 10. Influence des
précipitations journalières sur
les fluctuations de la densité du
bois initial de Pinus 3 et Pinus 4
(année 1995).

après l'observation de l'arrêt de la croissance [10, 36, 46]. Ensuite seulement, le protoplasme meurt et les trachéides sont complètement différenciées.

En ce qui concerne la confrontation des mesures en continu de la croissance avec les fluctuations intra-annuelles de la densité, certaines remarques doivent être faites sur la précision de l'approche. D'une part, l'ajustement profil - accroissements cumulés n'a pas tenu compte de la formation du phloème, et l'analyse densitométrique n'a pas la précision d'une approche cellulaire [45]. Il est donc difficile d'associer une échelle temporelle précise au profil annuel du cerne. D'autre part, cette difficulté est accentuée par les différences d'épaisseur notées pour un même cerne selon qu'il provient de l'analyse densitométrique ou du suivi en continu de la croissance. La dilatation (graphique) des profils nuit également à la précision. De plus, comme exposé précédemment, la non prise en compte des phénomènes de lignification, ayant une influence certaine sur les densitogrammes, peut réduire la précision et limiter l'information issue de la mise en relation entre la dynamique de croissance et les fluctuations de densité.

Ces différents points définissent les limites d'une telle approche. Une grande partie des faiblesses de la méthode pourrait être contournée par des mesures et des analyses réalisées en parallèle (suivi phénologique, flux de sève, ou bien analyse cellulaire...).
Les observations effectuées devront donc être interprétées avec précaution. Cependant, cette analyse a permis de rattacher, sans que l'échelle temporelle soit précise, les fluctuations de densité aux différentes étapes de la mise en place du cerne et donc globalement à la dynamique de croissance.

Autour d'une structure et d'un fonctionnement type, les variations d'un individu à l'autre reflètent les conditions micro-environnementales et les caractéristiques génétiques de l'individu, tandis que les variations interannuelles peuvent être mises en relation avec les fluctuations inter-annuelles du climat.

L'observation des variations inter-individuelles, semble mettre en évidence d'une part l'effet de la densité du peuplement, et d'autre part l'effet de l'âge, sur la croissance des quatre pins. Les deux pins ayant poussés au sein du peuplement (P3, P4) ont une croissance moindre que les deux pins ayant poussés en lisière (P1, $\mathrm{P} 2$ ). Cette observation illustre les phénomènes de compétitions au niveau des réserves hydrique et trophiques du sol, ainsi que les phénomènes de compétition pour la lumière. Des deux pins ayant poussés en lisière, le plus jeune possède la plus forte croissance. Il est cependant difficile de tirer des conclusions à partir de seulement quatre individus, de plus d'âge différent. L'étude des variations interindividuelles nécessitera la mise en place 
d'un protocole avec des arbres de statut et d'âge équivalent.

Les observations effectuées sur les concordances entre climat hivernal et démarrage de la croissance semblent montrer que c'est la combinaison des précipitations et des températures, plus que les seules températures, qui détermine le démarrage de la croissance. Il serait favorisé par un hiver doux et humide, et au contraire retardé par un hiver froid et sec. Cependant, des températures basses, indépendamment des précipitations, sont susceptibles de retarder fortement le démarrage de la croissance, surtout durant le mois de février. La succession d'une séquence chaude (janvier 1993) et froide (février 1993) est susceptible d'amplifier l'impact des basses températures. Ces observations expriment l'effet rémanent des températures hivernales basses sur la photosynthèse, et notamment les déphasages existant entre l'impact de basses températures et la reprise de la photosynthèse [15]. L'analyse des fonctions de réponses ne met pas en évidence ce type d'influence chez le pin d'Alep [30]. Les résultats obtenus sur l'influence des températures hivernales concernaient exclusivement l'impact de l'hiver 1956 qui a eu un effet traumatique répercuté sur plusieurs années (cernes absents).

L'analyse du comportement estival montre que, contrairement à ce qu'une simple observation pourrait laisser croire, les faibles précipitations estivales (période juin-juillet) ne constituent pas le facteur principal de la mise en repos estival. C'est la répartition des précipitations durant la période de végétation, et plus particulièrement l'importance des précipitations en avril-mai, qui semble déterminer le comportement estival du pin d'Alep. Cette observation met en évidence l'importance du bilan hydrique $[6,13,22]$ sur le comportement estival du pin d'Alep. La construction du cerne durant l'été est donc dépendante des précipitations qui renouvellent le stock d'eau dans le sol, de l'intensité de l'évapotranspiration déterminée par les températures et des caractéristiques édaphiques du sol, notamment la réserve utile. Ce phénomène a déjà été étudié en région méditerranéenne, notamment sur Pinus pinea, avec la mise en évidence de l'influence du paramètre ETP/ETR sur les variations inter-annuelles de la croissance [5]. Nos résultats montrent, par ailleurs, que le stress hydrique serait un déclencheur de la transition bois initial-bois final. Il est cependant difficile de déterminer la part relative du déterminisme climatique et du déterminisme interne ou phénologique pour cette étape de la construction du cerne. L'importance du bilan hydrique sur le comportement des peuplements forestiers n'est pas seulement réservée aux seuls peuplements forestiers méditerranéens.
On retrouve la même importance du bilan hydrique pour des espèces plus septentrionales, qui sont soumises à des sécheresses estivales nettement moins importantes [6, $22,23]$.

La présence d'une période de repos est souvent observée dans des régions soumises à une sécheresse estivale $[10,29]$. Elle exprime, chez le pin d'Alep, une stratégie d'adaptation vis-à-vis de l'important stress hydrique. Il régule ainsi de façon efficace ces pertes transpiratoires [3] et évite les risques d'embolie estivale [2].

L'occurrence de précipitations suite à la période de sécheresse est responsable d'une reprise de la croissance. Ce phénomène de reprise, faisant suite à une période de repos, est responsable des fluctuations de densité au sein du bois final. L'occurrence de précipitation a un effet direct sur la balance hydrique de l'arbre qui devient nettement positive. Les cellules deviennent alors turgescentes, ce qui favorise l'élongation cellulaire [17, 7]. Il en résulte la formation de cellules à lumen plus grand. Ce même processus concerne également «l'événement ponctuel » noté au sein du bois initial. Dans le cas du bois final, les cellules ne retrouvent cependant pas l'aspect des cellules du bois initial, l'épaisseur des parois restant importante.

En fonction de l'augmentation plus ou moins importante de l'élongation cellulaire et de la diminution de l'épaisseur des parois, les fluctuations de densité seront plus ou moins visibles sur le faciès du cerne. Dans le cas où elles sont nettement visibles, on a affaire à ce qu'on appelle un cerne double [16], phénomène fréquent chez le pin d'Alep [35, 41]. Ces processus, dans les cas extrêmes, associés à une reprise de la croissance des pousses et ou des aiguilles $[19,20]$, sont responsables de la formation d'un faux cerne, donnant l'impression d'un cerne additionnel apparemment complet [16, 36].

La réalité d'une période de repos, pourtant bien marquée, peut cependant être remise en question. En effet, on observe en 1993, dans le cas de Pinus 1 une période de repos mais pas ou peu de fluctuations de densité au sein du bois final. La croissance, très faible durant la période estivale, pourrait être alors masquée par le rétrécissement du tronc $[9,11]$. Il est donc possible que l'activité cambiale ne soit pas complètement stoppée mais seulement fortement ralentie. Seule, une analyse cellulaire simultanée avec le suivi en continu de la croissance permettrait de préciser, s'il y a effectivement une période de repos.

En ce qui concerne l'arrêt de la croissance nous n'avons que très peu d'observations. Cependant, on peut noter que, la moyenne des quinze premiers jours 
d'octobre étant de $14{ }^{\circ} \mathrm{C}$, la température ne semble pas être responsable de l'arrêt de la croissance. Le déterminisme de cet arrêt serait à chercher au niveau des potentialités annuelles du cambium en liaison avec les autres processus internes (mise en réserves, initiation des bourgeons, rhizogénèse...).

\section{CONCLUSION}

Malgré les aléas liés à la méthode elle-même et ses contraintes techniques, cette approche apporte des enseignements proches du fonctionnement même de l'arbre et donc des relations de cause à effet. Certains des résultats obtenus mettent en évidence des relations entre la croissance radiale et le climat qui ne peuvent pas être révélées par les seules fonctions de réponses. De tels résultats sont donc complémentaires de ceux obtenus par les approches statistiques classiques. De plus, la mise en évidence des différentes phases d'élaboration du cerne et de leur relation avec les fluctuations intra-annuelles de densité permettra une interprétation plus aisée des résultats obtenus avec les approches statistiques, notamment en replaçant les différentes composantes structurelles du cerne dans le temps.

La prise en compte de la variable explicative climat a montré le rôle majeur des précipitations sur les variations inter-annuelles des rythmes et des taux de croissance, ainsi que sur les fluctuations de la densité. On a pu mettre en évidence une influence des précipitations sur des séquences temporelles allant de l'événement climatique à des séquences pluri-mensuelles. L'exploitation des profils densitométriques constitue une autre voie d'accès à la compréhension des relations climat-croissance-structure du cerne. Les profils de densité sont de meilleurs enregistreurs de la répartition des précipitations et des événements climatiques ponctuels que ne le sont les épaisseurs de cernes. L'utilisation des profils de densité serait donc très utile pour l'interprétation des années caractéristiques [38] dans les approches de type dendroécologiques.

D'une façon générale, il ressort de ce travail la très forte influence des séquences temporelles qui précèdent la période estivale, et donc du bilan hydrique sur la croissance radiale du pin d'Alep. Ces résultats confirment l'intérêt, déjà soulevé par certains auteurs [5, 13], de mettre en œuvre des paramètres de types bioclimatiques ou écophysiologiques dans la modélisation des relations croissance-climat. Ce type d'approche permettrait de mieux appréhender le fonctionnement de l'arbre et de définir plus précisément le rôle du biotope dans la relation entre croissance et climat.

Remerciements : Nous remercions tout spécialement Kurt Loris pour ses remarques et son aide précieuse dans l'installation de la station de mesure.

\section{RÉFÉRENCES}

[1] Alifriqui M., M'Hirit O., Peltier J.P., Tessier L., Analyse comparée de la croissance radiale du pin d'Alep et du Cyprès de l'Atlas dans le Haut Atlas Occidental Marocain, Dendrochronologia 14 (1996) 71-87.

[2] Attolini M. R., Calvani F., Galli M., Nanni T., Ruggiero L., Schar E., Zuanni F., The relationship between climatic variables and wood structure in Pinus halepensis Mill. Effect of humidity and temperature, Theor. Appl. Climatol. 41 (1990) 121-127.

[3] Aussenac G., Valette J.C., Comportement hydrique estival de Cedrus atlantica Maneti, Quercus ilex L., Quercus pubescens Willd. et de divers pins dans le Mont Ventoux, Ann. Sci. For. 39 (1982) 41-62.

[4] Bertaudière V., Montes N, Gauquelin T., Édouard J.L., Dendroécologie du genévrier thurifère (Juniperus thurifera L.) : exemple de la thuriféraie de la montagne de Rié (Pyrénées, France), Ann. For. Sci. 56 (1999) 685-697.

[5] Boreux J.J., Gadbin-Henry C., Guiot J., Tessier L., Radial tree-growth modelling with fuzzy regression, Can. J. For. Res. 28 (1998) 1249-1260.

[6] Bréda N., Bilan hydrique et impact des épisodes de sécheresse sur la croissance radiale des chênes. Dépérissement forestier en vallée du Rhin, Rapport scientifique final convention ONF/INRA, 1994, 60p.

[7] Catesson A.M., Cambial Cytology and Biochemistry, in: Iqbal M. (Ed.) The Vascular Cambium, 1990, pp. 63-112.

[8] Creber G.T., Chalonner W.G., Environmental influences in cambial activity, in: Iqbal M. (Ed.) The Vascular Cambium, 1990.

[9] Fritts H.C., An analysis of radial growth of beech in a central Ohio forest during 1954-1955, Ecology 39 (1958) 705-720.

[10] Fritts H.C., Tree ring and climate, Academic Press, New York, 1976.

[11] Fritts H.C., Smith D.G., Stokes M.A., The biological model for paleoclimatic interpretation of Mesa Verde tree-ring series, Amer. Antiq. 31 (1965) 101-121.

[12] Granier A., Bréda N., Modelling canopy conductance and stand transpiration of an oak forest from sap flow measurement, Ann. Sci. For. 53 (1996) 537-546.

[13] Granier A., Bréda N., Biron P., Vilette S., A lumped water model to evaluate duration and intensity of drought constraints in forest stands, Ecol. Model. 116 (1999) 269-283. 
[14] Green H.V., Worall J., Wood qualities studies. 1- Scanning microphotometer for measuring and recording certain wood characteristics. Pulp and paper Res. Inst. Canada, Tech. Rep. 1963.

[15] Guehl J.M., Étude comparée des potentialités hivernales d'assimilation carbonée de trois connifères de la zone tempérée (Pseudotsuga menziesii Mirb., Abies alba Mill. et Picea excelasa Link.), Ann. Sci. For. 42 (1985) 23 -38.

[16] Kaennel M., Schweingruber F.H., Multilingual glossary of dendrochronologie, WSL/FNP. Haupt Publishers, Berne, Stuttgart, Vienna, 1995.

[17] Kozlowski T.T., Kramer P.J., Pallardy G., The physiological ecology of woody plants, Academic Press, San Diego, 1991.

[18] Kozlowski T.T., Winget C.H., Diurnal and seasonnal variation in radii of tree stems, Ecology 45 (1964) 149-155.

[19] Larson P.R., A physiological consideration of the springwood summerwood transition in red pine, Forest Sci. 6 (1960) 110-122.

[20] Larson P.R., Auxins gradients and the regulation of cambial activity, in: Kozlowski T.T. (Ed.), Tree Growth, Ronald Press, New York, 1962, pp. 97-117.

[21] Larson P.R., Wood formation and the concept of wood quality, Bulletin No. 74, School of forestry and environnemental studies, Yale University, New Haven, 1969.

[22] Lebourgeois F., Analyse du bilan hydrique et de la croissance des arbres dans le RENECOFOR. Rapport scientifique final, Union Européenne, DG VI, projet INRA-CEE, 1999.

[23] Lebourgeois F., Climatic signal in earlywood, latewood and total ring width of Corsican pine from western France, Ann. For. Sci. 57 (2000)155-164.

[24] Lens O., Schar E., Schweingruber F.H., Methodische Probleme bei der radiographisch-densitometrischen Bestimmung der Dichte und der Jahrringbreiten von Holz, Holzforschung 30 (1976) 114-123.

[25] Liphschitz N., Lev-yadun S., Rosen E., Waisel Y., The annual rhythm of activity of the lateral meristem (cambium et phellogen) in Pinus halepensis Mill. and Pinus pinea L., IAWA Bull. 5 (1984) 263-274.

[26] Liphschitz N., Lev-yadun S., Cambial activity of evergreen and seasonal dimorphic around the Mediterranean, IAWA Bull. 7 (1986) 145-153.

[27] Liphschitz N., Mendel L., Comparative radial growth of Pinus halepensis Mill. and Pinus brutia in Israël, Forêt Méditerranéenne 9 (1987) 115-117.

[28] Loris K., Dickenwachstum von Zirbe; Fichte und Lärche an der alpinene Wald-Grenze/Patscherkofel. Ergebnisse der Dendrometermessungen 1976/79, Mitteilungen der forstlichen Bundesversuchsanstalt Wien, 142 (1981) 417-441.

[29] Loris K., Methoden der permanent - Zuwachsemenssung. Seminar der Bayer. Landesanstalt für Wald und Forstwirtschaft. Schriftenreihe der foorstwissenschaftlichen Fakultät der Universität München und der Bayer-Landesanstalt für Wald und Forstwirtschaft, 1995.
[30] Nicault A., Analyse de l'influence du climat sur les variations inter- et intra-annuelles de la croissance radiale du pin d'Alep (Pinus halepensis Mill.) en Provence calcaire. Thèse de l'Université d'Aix-Marseille III, 1999. 254 p.

[31] Parker M.L., Dendrochronological techniques used by the geological survey of Canada, in: Smith J.H.G. and Worall (Eds.), Trees ring analysis with special reference to western North America. Faculty of Forestry, Bulletin No. 7, University of British Columbia, Vancouver, 1970.

[32] Polge H., L'analyse densitometrique de clichés radiographiques : une nouvelle méthode de la détermination du bois, Ann. École Eaux Forêts Station Recherche et Expériences 20 (1963) 530-581.

[33] Polge H., Établissement des courbes de variations de la densité du bois par exploration densitométrique de radiographies d'échantillons prélevés à la tarrière sur des arbres vivants. Applications dans les domaines techniques et phytosociologiques, Ann. Sci. For. 23 (1966) 1-206.

[34] Rathgeber C., Nicault A., Keller T., Guiot J., Roche P., Guibal F., Pinus halepensis forests Production responses to climate and atmospheric change as simulated by a statistical model, Global and planetary change 26 (2000) 405-421.

[35] Safar W., Contribution à l'étude dendoécologique du pin d'Alep (Pinus halepensis Mill) dans une région semi-aride d'Algérie : l'Atlas Saharien (Ouled Naïl-Aurès-Hodna), Thèse de l'université d'Aix-Marseille III, 1994.

[36] Schweingruber F. H., Tree rings. Basic and application of dendrochronology, D. Reidel publishing company, Dordrecht, 1988, p. 276.

[37] Schweingruber F. H., Radiodensitometry, in: Cook E.R \& Kairiukstis L.A. (Eds.), Methods of dendrochronology, Applications in Environmental Sciences, Kluwer Acad. Publ., Dordrecht, 1990, pp. 55-63.

[38] Schweingruber F. H., Eckstein D., Serre-Bachet. F., Bräker O. U., Identification, presentation and interpretation of event years and pointer years in dendrochronology, Dendrochronologia 8 (1990) 9-37.

[39] Serre F., Contribution à l'étude dendroclimatologique du pin d'Alep (Pinus halepensis Mill.). Thèse, Université d'AixMarseille III, 1973.

[40] Serre-Bachet F., Les rapports de la croissance et du climat chez le pin d'Alep (Pinus halepensis Mill). I - Méthodes utilisées. L'activité cambiale et le climat, Oecol. plant. 11 (1976) 143-171.

[41] Serre-Bachet F., Les enseignements écologiques de la variation de l'épaisseur du cerne chez le pin d'Alep, Forêt Méditerranéenne 13 (1992) 171-176.

[42] Serre-Bachet F., Tessier L., Loris K., Mise en place et signification du cerne, in: T. Hackens, A.V. Munaut and Cl. Till (Eds.), Wood and Archeologie, P.A.C.T. Revue, First European Conference, October 2nd-3rd 1987, Louvain-la-Neuve, 1988.

[43] Serre-Bachet F., Tessier L., Response function analysis for ecological study, in: Cook E.R. and Kairiukstis L.A. (Eds.), Methods of dendrochronology, Applications in Environmental 
Sciences, Kluwer Acad. Publ. Dordrecht, Dordrecht, 1990, pp. 247-258.

[44] Tyree M.T., Cochard H., Summer and winter embolism in oak: impact on water relations, Ann. Sci. For. 53 (1996) 173-180.

[45] Vaganov E.A., The tracheidogram method in tree-ring analysis and its application, in: Cook E.R. and Kairiukstis L.A. (Eds.), Methods of dendrochronology, Applications in Environmental Sciences, Kluwer Acad. Publ. Dordrecht, Dordrecht, 1990, pp. 63-76.
[46] Wardrop A.B., The structure and formation of the cell wall in xylem, in: Zimmermann M.H. (Ed.), The formation of wood in forest trees, Academic Press, New York, London, San Francisco, 1964, pp. 87-134.

[47] Wilson B.F., Wodzicki T.J., Zahner R., Differentiation of cambial derivatives: Proposed terminology, Forest. Sci. 12 (1966) 438-440.

[48] Young H.E., Pratical limitations of the dial gauge dendrometer, Ecology 33 (1952) 568-570.

To access this journal online: www.edpsciences.org 